\title{
Market impact analysis of China A-shares into MSCI \\ index--comparison of volatility between MSCI China international
}

\section{index and CSI 300 index}

\section{Lu Gao *, Lian Chen}

School of Economic \& Management Nanjing University of Science \&Technology

*Corresponding author: Lu Gao, postgraduate, 379279014@qq.com

\begin{abstract}
:
In June 21, 2017, MSCI officially announced that from the beginning of June 2018 ,China A-shares will be included in the MSCI emerging markets index and MSCI ACWI (All Country World Index) global index. The significance of this paper lies in: through the comparison of the relevant index's volatility ,from the results of empirical research, we analyzed the domestic investors investment behavior, explained the significance of China's A-share index into MSCI index, and put forward the corresponding suggestions of the development of Chinese securities market.
\end{abstract}

Key words: MSCI, China A-share international index, CSI 300 index ,volatility,GARCH model

\section{Introduction}

Since 2002 Chinese capital market introduced QFII system, China capital market has been strengthening the degree of opening up gradually. From the implementation of QFII and RQFII system, liberalization of foreign institutions to invest in the interbank bond market, to Shanghai-Hong Kong Stock Connect program, Shenzhen-Hong Kong Stock Connect program has officially set sail. A series of opening measures have made great changes in China's capital market,and the international influence has been continuously improved. Since then,China A-shares will be included in the MSCI emerging markets index in 2018.Its initial accession of 222 A-shares of large cap stocks accounted for about $0.73 \%$ of the index weight, which means that China's financial market opening to the outside world entered a new stage. 


\section{Analysis of MSCI China's A-share Index Volatility}

\subsection{Samples and data}

We choose MSCI Chinese A-share international stock index and CSI 300 index daily closing value as the sample data, the sample data of the period range from January 4, 2016 to July 31, 2017,excluding stock exchange holidays,there are 399 samples.MSCI Chinese A-share international stock index reflects investment opportunities for Chinese A-shares available for purchase from the perspective of international QFII/RQFII investors, which is the index that MSCI emerging markets will eventually be absorbed by. Therefore, we select MSCI Chinese A-share international stock index(the following is abbreviated as MSCI index) and CSI 300 index to compare and observe.

\subsection{General description of data}

The data used in this paper are from\ Wind database, and use Eviews software to give the results of the relevant data processing.RM stands for MSCI index yield, RA represents the CSI 300 yield.

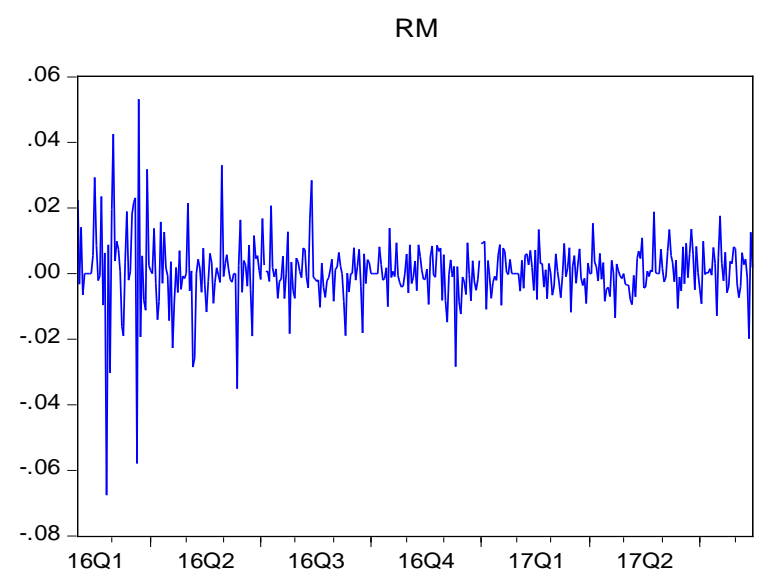

Fig. 1 - The daily yield sample of MSCI

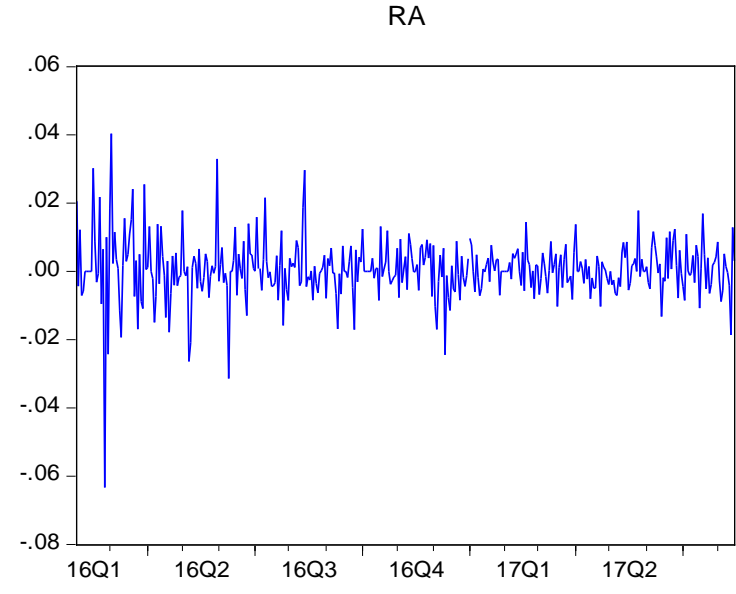

Fig. 2 - The daily yield sample of CSI 300 index

Fig. 1 and Fig. 2 are the time series on the yield of MSCI index and CSI 300 index, based on the description of the index changes, we can preliminary judge the volatility of MSCI index and CSI 300 index. Through comparison of Fig. 1 and Fig. 2, we can see that from the first quarter of 2016 to the second quarter of 2016,two index fluctuations are both relatively large, but during this period, fluctuation of MSCI index is more volatile than CSI 300 index .From the third quarter of 2016 to the second quarter of 2017, both fluctuated, but the fluctuation is small. In addition we can observe the logarithmic rate of return volatility "cluster" 
phenomenon. It can be seen from the figure obviously "clustering", namely big (small) after fluctuations tend to large (small) fluctuations. The "heavy tail" features can also be observed from the figure.

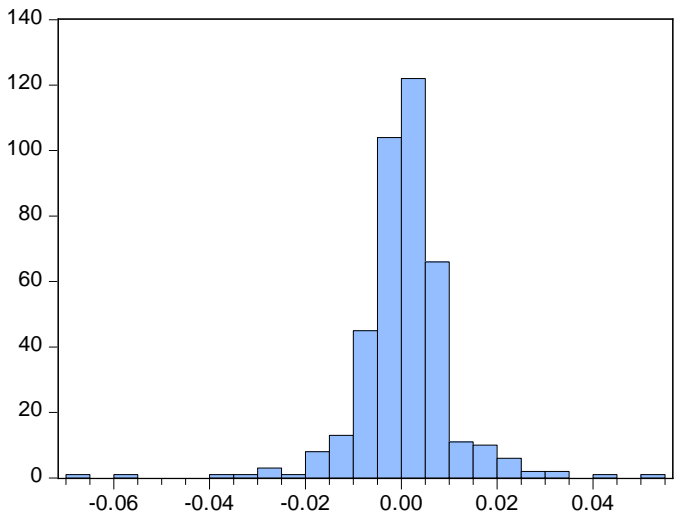

$$
\begin{array}{|lr|}
\hline \multicolumn{2}{|l|}{\text { Series: RM }} \\
\text { Sample 2/02/2016 8/15/2017 } \\
\text { Observations } 399 \\
\text { Oean } & 0.000538 \\
\text { Median } & 0.000000 \\
\text { Maximum } & 0.053176 \\
\text { Minimum } & -0.067555 \\
\text { Std. Dev. } & 0.010180 \\
\text { Skewness } & -0.700929 \\
\text { Kurtosis } & 13.21235 \\
& \\
\text { Jarque-Bera } & 1766.528 \\
\text { Probability } & 0.000000
\end{array}
$$

Fig. 3 - Histogram of MSCI Chinese A-share international stock index yield

Fig. 3 is a general description of the data of MSCI index. In Fig. 3 can be seen from 2016 to 2017,the mean value of the selected 399 samples is 0.000538,skewness is -0.700929 , indicating the left deviation, kurtosis is 13.21235 ,which shows that the return distribution is far more than just the normal distribution of "peak".Jarque-Bera statistics of the corresponding $\mathrm{p}$ value is 0 , means that the probability of yield obeying normal distribution is almost 0 , return sequence distribution is significantly different from normal distribution.
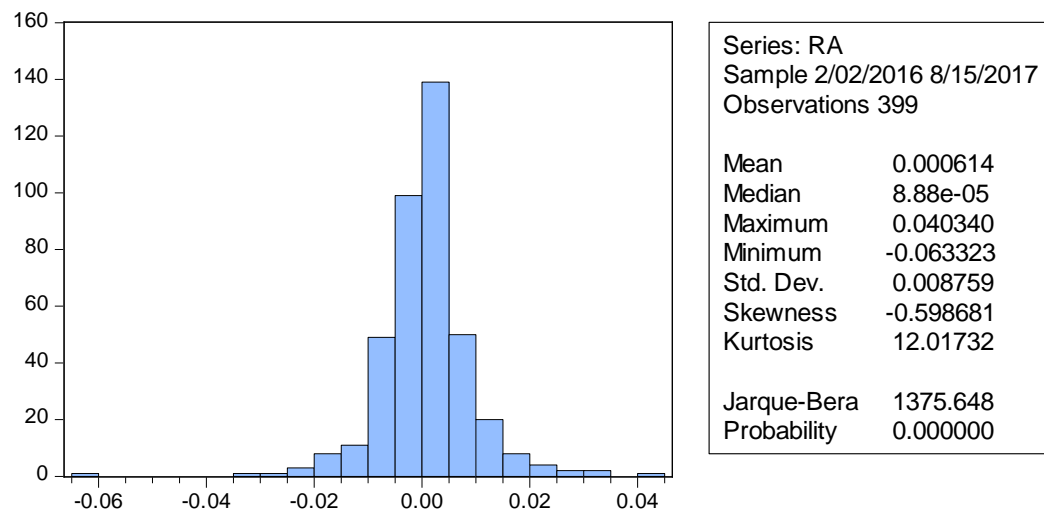

Fig. 4 - Histogram of CSI 300 index yield

Fig. 4 is a general description of the data of CSI 300 index. From Fig. 4 we can see that from 2016 to 2017, the mean value of the selected 399 samples is 0.000614 , skewness is -0.598681 ,indicating the left deviation,kurtosis is 12.01732 , shows that the return distribution is far better than the normal distribution of "peak".Jarque-Bera statistic corresponding to the value of $\mathrm{P}$ is 0 ,means that the probability of yield obeying normal distribution is almost 0 , 
return sequence distribution is significantly different from normal distribution.

\subsection{ARCH effect test}

In order to test the ARCH effect of MSCI index and CSI 300 index and confirm the stationarity of the return series, the ADF unit root test for the logarithmic closing value of the index is given. The results are given by Table1 and Table 2 as below:

Null Hypothesis: RM has a unit root

Exogenous: Constant

Lag Length: 0 (Automatic based on SIC, MAXLAG=16)

Table 1 - Stability test of MSCI index yield

t-Statistic Prob.*

Augmented Dickey-Fuller test statistic $\quad-23.00540 \quad 0.0000$

Test critical values: $1 \%$ level

$-3.446567$

$5 \%$ level

$-2.868583$

$10 \%$ level

$-2.570588$

According to Table1, the ADF statistic of the yield series is -23.00540 , Prob is 0.0001 , and is significant at the level of $1 \%$. The hypothesis of rejecting random walk can be considered that the return index sample sequence is stable.

Null Hypothesis: RA has a unit root

Exogenous: Constant

Lag Length: 0 (Automatic based on SIC, MAXLAG=16)

Table 2 - Stability test of CSI 300 yield

\begin{tabular}{lccc}
\hline \hline & t-Statistic & Prob.* \\
\hline \hline Augmented Dickey-Fuller test statistic & -20.20001 & 0.0000 \\
\hline Test critical values: & 1\% level & -3.446567 & \\
& $5 \%$ level & -2.868583 & \\
& $10 \%$ level & -2.570588 & \\
& & \\
\hline \hline
\end{tabular}


From Table 2, the ADF statistic yield sequence is -20.20001 , Prob is 0.0001 , significant at $1 \%$ level. The random walk hypothesis can be rejected, that income index of the samples with a smooth sequence. Thus we can determine the rate of return of RM and RA were stable financial time series.

We made a heteroscedasticity test of the yield, which is the ARCH effect test. The main idea is that in the time series data, the heteroscedasticity can be considered as a ARCH process, and whether the time series has heteroscedasticity can be determined by testing whether the process is established.If there is heteroscedasticity,that is,the ARCH effect, then the GARCH model should be used for analysis. As long as Probability is less than 0.05, is that there is ARCH effect. From the results, RM ARCH (1) (a lag period) Probability is greater than 0.05 ARCH (4) (lag four) Probability is less than 0.05, indicating the existence of ARCH effect, that is, there is heteroscedasticity. The Probability of ARCH (1) is greater than 0.05 in RM,but the Probability of ARCH(4) were less than 0.05,indicating the presence of ARCH effect, namely heteroscedasticity. Generally considered whether there is ARCH effect test, ARCH 3-5 phase lag is more appropriate, the judgment result is accurate.

Table 3 - ARCH effect test

\begin{tabular}{|c|c|c|c|c|c|c|c|c|}
\hline & \multicolumn{4}{|c|}{ ARCH (1) } & \multicolumn{3}{c|}{ ARCH (4) } \\
\hline \multirow{2}{*}{ series } & F-statisti & Pro. & $\mathrm{T}^{*} \mathrm{R}^{2}$ & Pro. & $\begin{array}{c}\text { F-statisti } \\
\text { c }\end{array}$ & Pro. & $\mathrm{T}^{*} \mathrm{R}^{2}$ & Pro. \\
\hline RM & 13.882 & 0.0002 & 13.491 & 0.002 & 7.482 & 0.000 & 28.145 & 0.000 \\
\hline RA & 0.223 & 0.6364 & 0.223 & 0.6354 & 7.303 & 0.000 & 27.519 & 0.000 \\
\hline
\end{tabular}

By Table 3, two index has the ARCH effect, which are heteroscedasticity. Next, the GARCH model is adopted.

\section{3 the Empirical Analysis,Results and Discussion}

First, the mean equation is determined. According to the most commonly used model selection criteria, the parameter estimation is significant. Secondly, the smaller the AIC and SBC values, the bigger the $\mathrm{R}^{2}$ value,the result is better. MSCI Chinese A-share international stock average index equations of optimal order is 1 . Then to determine the order variance equation $\mathrm{ARCH}$ and $\mathrm{GARCH}$, through the comparison of simulation results, we select GARCH $(1,1)$ of the MSCI index . In order to reflect that since March 2017 the stock market gradually released the news of China joining MSCI signal on the influence of volatility, we add a variable D01, D01=0 said before March 2017 and after March 2017, D01=1 said. 


\subsection{Risk premium effect}

The result of MSCI index GARCH-M model is:

$$
\begin{aligned}
\sigma_{t}^{2}= & 1.28 \times 10^{-6}+0.058566 \varepsilon_{t-1}^{2}+0.928542 \sigma_{t-1}^{2}-1.61 \times 10^{-7} D 01 \\
& (2.118889)(6.643577)(96.56630) \quad(-0.308585)
\end{aligned}
$$

$\operatorname{ARCH}\left(\varepsilon_{t-1}^{2}\right)$ and GARCH $\left(\sigma_{t-1}^{2}\right)$ were highly significant, and their significance was 0 , less than 0.05, indicating significant.D01 significance of 0.7576, indicating no significant. The result of CSI 300 index GARCH-M model is:

$$
\begin{aligned}
\sigma_{t}^{2}= & 6.42 \times 10^{-7}+0.044975 \varepsilon_{t-1}^{2}+0.944789 \sigma_{t-1}^{2}+1.25 \times 10^{-7} D 01 \\
& (1.240231)(5.540184)(95.91577) \quad(0.301079)
\end{aligned}
$$

$\operatorname{ARCH}\left(\varepsilon_{t-1}^{2}\right)$ and GARCH $\left(\sigma_{t-1}^{2}\right)$ were highly significant, and their significance was 0 , less than 0.05, indicating significant.D01 significance of 0.7634, indicating no significant.MSCI index and CSI 300 investors' risk aversion parameters were 0.058566 and 0.044975 respectively, and significant at the level of 5\%, which shows that the two markets have a significant risk reward, high risk require high return, the volatility increasing the earnings expectations of investors. In addition, the MSCI index of the risk premium is higher than the CSI 300 index, MSCI index shows that investors are more risk averse, with higher risk compensation. From the other side ,it also shows that the idea of investor investment in China's stock market needs to be improved.

\section{2 the leverage effect analysis}

The EGARCH $(1,1)$ model is adopted,and the result of MSCI index is:

$$
\log \left(\sigma_{t}^{2}\right)=-17.53331+0.321739\left|\frac{\varepsilon_{t-1}^{2}}{\sqrt{\sigma_{t-1}^{2}}}\right|-0.055438 \frac{\varepsilon_{t-1}^{2}}{\sqrt{\sigma_{t-1}^{2}}}-0.859880 \log \left(\sigma_{t-1}^{2}\right)-1.038328 \mathrm{D} 01
$$

The $\mathrm{Z}$ values corresponding to each coefficient were $-102.9451,8.439886,-2.842784,-41.07678$, -3.853208., and their significance was less than 0.05, indicating significant.

The EGARCH model shows the existence of the same energy "bad" brought about by the fluctuation is much larger than the "good news" caused by the fluctuations. The coefficient of ARCH in EGARCH model is 0.321739 , greater than 0 .The coefficient of the asymmetric term is 0.055438 , which is negative. Both are significant, showing the information asymmetry effects, exists the leverage effect in the sample.When the good news, there is a $0.321739-0.055438=0.267359$ impact; and the emergence 
of "bad news", it will bring $0.321739+0.055438=0.377177$.That is to say, the market is more responsive to bad news. In addition, we also found that GARCH $(1,1)$ D01 virtual model is not significant in this model is significantly positive, that in the news release, the MSCI index fluctuates more dramatically.

The result of CSI 300 index EGARCH is:

$\log \left(\sigma_{t}^{2}\right)=-0.059526+0.601410\left|\frac{\varepsilon_{t-1}^{2}}{\sqrt{\sigma_{t-1}^{2}}}\right|-0.087783 \frac{\varepsilon_{t-1}^{2}}{\sqrt{\sigma_{t-1}^{2}}}+0.998411 \log \left(\sigma_{t-1}^{2}\right)+0.010628 D 01$

The coefficients of the corresponding $Z$ values were: -1.659272,3.139416, -5.759143,244.4909,1.622309.The $\left|\frac{\varepsilon_{t-1}^{2}}{\sqrt{\sigma_{t-1}^{2}}}\right|, \frac{\varepsilon_{t-1}^{2}}{\sqrt{\sigma_{t-1}^{2}}}$ and $\log \left(\sigma_{t-1}^{2}\right)$ coefficient was significantly less than 0.05 significantly; the significance of the D01 is 0.1047 , more than 0.05 , not significant.The EGARCH model shows the existence of the same energy "bad" brought about by the fluctuation is much larger than the "good news" caused by the fluctuations.In the EGARCH model, the coefficient of ARCH is 0.60141, greater than 0,Non symmetric coefficient is 0.087783 , negative.The two coefficients were significant, indicating that the information asymmetry effects, that existing leverage effect in the sample. When favorable news, there is a $0.60141-0.087783=0.513627$ impact; while the emergence of "bad news", it will bring $0.60141+0.087783=0.689193$ shock. That is to say, the market reflects bad news more strongly.Besides, we also find that the virtual item D01 is not significant in this model.Specific comparison found that, whether it is good news, the impact of the MSCI index is greater than CSI 300 .

\subsection{Spillover effect analysis}

The result of MSCI index model is:

$\sigma_{t}^{2}=-1.24 \times 10^{-5}-0.012723 \varepsilon_{t-1}^{2}+0.512200 \sigma_{t-1}^{2}+0.336868 \sigma_{t-1 \text { CSI } 300}^{2}+1.78 \times 10^{-6} D 01$

The $\mathrm{Z}$ value corresponding to each coefficient are: $-1.944918,-0.540020$, 2.075770,1.403572,0.515425.The $\sigma_{t-1}^{2}$ was significantly less than 0.05 ,showing positive, the remaining variables were not significant. $\sigma_{t-1 C S I 300}^{2}$ interference in CSI 300 index of MSCI index, if positive, showing that there is a significant spillover effect from the Chinese market to the MSCI index market. But this study shows that $\sigma_{t-1 C S I 300}^{2}$ is not significant,the spillover 
effect of China's stock market on the MSCI index market does not exist. D01 variable in this study is also not significant.

The result of CSI 300 index model is:

$$
\sigma_{t}^{2}=-1.87 \times 10^{-6}+0.016337 \varepsilon_{t-1}^{2}+0.679876 \sigma_{t-1}^{2}+0.832314 \sigma_{t-1 \mathrm{MSCI}}^{2}+1.11 \times 10^{-6} \mathrm{D} 01
$$

The coefficients of the corresponding $Z$ values were: $-0.768373,0.669718$, 2.986184,2.189716,0.571831.The $\sigma_{t-1}^{2}$ and $\sigma_{t-1 \mathrm{MSCI}}^{2}$ was significantly less than 0.05 ,showing positive, the remaining variables were not significant. $\sigma_{t-1 \mathrm{MSCI}}^{2}$ stands for the interference of MSCI index to CSI 300 index, if positive, shows that from the MSCI index has spillover effects on Chinese local stock market .In this study, $\sigma_{t-1 \mathrm{MSCI}}^{2}$ is significantly positive, the spillover effect of MSCI index on the market Chinese stock market exists in the statistical sense. D01 in this study is still not significant.

From the empirical analysis we can see that,the asymmetry of market spillover effect between Chinese stock market and MSCI index shows that the two markets conduction is unilateral, MSCI index has certain demonstration effect on the Chinese stock market.

\section{Conclusions}

Considering the initial MSCI ratio and the size of capital inflows, investors in the short term are more emotionally charged for A-share market, investors' behavior may be shaped in the medium and long term. From the view of 222 stocks included in the MSCI,the medium market value will exceed 50 billion, and most of the sample stocks are blue chip stocks.As future MSCI adoption factors increase from $5 \%$ to full, these stocks will continue to have long-term buying funds. These advantages will help to construct and influent the long-term investment behavior and value concept of A-share stock investors.

\section{References}

1. Fei Han , Hui Xiao . Linkage analysis between Chinese and American stock markets [J]. financial research 11 (2005) 117-129

2. Tao Li. A comparative study on the volatility characteristics of the stock market across the Taiwan Straits under the process of financial integration [J]. Commercial research, 56 (7) (2014) 60-65. 
3. MinHsien Chiang, and JoYu Wang. "Regime switching cointegration tests for the Asian stock index futures: evidence for MSCI Taiwan, Nikkei 225, Hong Kong Hang-Seng, and SGX Straits Times indices." Applied Economics 40.3 (2008) 285-293.

4. Du Yulin. Research on the integration of emerging stock markets and developed stock markets -- An Empirical Analysis Based on the Korean stock index into the MSCI-EM index, [J]. price theory and practice 12 (2016) 131-134. 